\title{
Friendsourcing the Unmet Needs of People with Dementia
}

\author{
João Martins, José Carilho, Oliver Schnell, Carlos Duarte, Francisco M. Couto, Luís Carriço, \\ Tiago Guerreiro \\ University of Lisbon / LaSIGE \\ FCUL, Departamento de Informática, Edifício C6, Campo Grande \\ \{joao.martins, jose.carilho, oliver\}@lasige.di.fc.ul.pt, \{cad,fcouto,Imc, tjvg\}@di.fc.ul.pt
}

\begin{abstract}
The decay of cognitive abilities associated with dementia severely impacts the quality of life of a person and his surrounding ecosystem. First, people with dementia (PwD) increasingly forget the events in their lives leading to depression, isolation and faster cognitive deterioration. Second, their caregivers, besides the emotional burden that having someone close in such a condition carries, are also likely to be overwhelmed with responsibilities and duties in maintaining the PwD with a balanced and decent lifestyle. Our approach tries to address this by providing a prosthetic memory captured in two ways: 1) automatically captures data from the PwD's smartphone and enriches it with automatically retrieved data from the web and, 2) a private social network group (friendsourcing) used to validate and personalize the relevant events in the PwD's life. By sharing the load among a limited but still populated set of trustworthy people we aim to maintain the caregiving process feasible and maintain more people engaged with the PwD. Overall, the ultimate goal is to provide reminiscence, safety and cognitive aids for the PwD that are up-to-date and personally relevant.
\end{abstract}

\section{Categories and Subject Descriptors}

H.5.m. Information interfaces and presentation (e.g., HCI): Miscellaneous.

\section{Keywords}

Dementia, Cognitive Prosthesis, LifeLogging, Friendsourcing.

\section{INTRODUCTION}

As trends in life expectancy increase, the number of age-related impairments, physical and cognitive, and risk of chronic disease within the older population is expected to rise. Around the globe, it is estimated that more than 35 million people have dementia. Along, this is expected to reach more than 115 million within the next 40 years [1]. Dementia is often translated in memory problems, difficulties in communicating, maintaining a healthy routine, and an increasing lack of perceived safety that ultimately leads to more isolation and diminished social contact.

Permission to make digital or hard copies of all or part of this work for personal or classroom use is granted without fee provided that copies are not made or distributed for profit or commercial advantage and that copies bear this notice and the full citation on the first page. To copy otherwise, or republish, to post on servers or to redistribute to lists, requires prior specific permission and/or a fee.

W4A2014 - Communication, April 7-9, 2014, Seoul, Korea. Co-Located with the 23nd International World Wide Web Conference. Copyright 2014 ACM 978-1-4503-2651-3...\$15.00.
Advances in mobile computing systems, sensing mechanisms and communications, both at home and when mobile, enabled the deployment of tools to improve the quality of life (QoL) of people with dementia (PwD). In fact, current mobile devices gather rich sensing and processing abilities to help the user in recognizing his environment and comprise communication abilities that allow remote communication with caretakers and clinical bodies. Further, they also include input and output mechanisms, along with interfaces that, adapted the right way, allow for a richer and more natural way for elderly users to interact with.

Despite this, most approaches to support memory, increase social contact and enhance feelings of safety, still fall short showing very low deployment rates. Among other reasons, most approaches seek to provide local solutions to specific needs, failing to consider the whole eco-system around the person with mild dementia. Further, current tools to address the needs of people with Alzheimer place the load in a very restricted set of informal caregivers limiting the success of those approaches and leading those people to excruciating conditions [13].

Our approach to tackle the unmet needs of PwD is based on gathering autobiographical data automatically [11] using mainstream smartphones towards rich cognitive prosthesis. However, we call for a paradigm shift where the PwD is placed in a center of a larger support network than what is seen in most cases nowadays. To do so, we built a system that although initially fed automatically uses a private social network to validate, enrich and give relevance to the events in the person's life. With this approach we seek to provide more accuracy to the data in the cognitive prosthesis but also, in parallel, to maintain a larger group of people engaged with improving the QoL of the PwD.

\section{RELATED WORK}

The top unmet needs of PwD can be categorized as follows: support for memory - reminiscing and reminding; support for social contacts - enable communication with family and friends; support with daily activities - Help executing recreational and useful activities; enhance feelings of safety - Prevent people with dementia from experiencing anxious or dangerous situations [16]. Ultimately, ICT tools seek to meet one or more of the aforementioned needs. Next, we summarize related work in those areas as well as in friendsourcing, a pillar to our approach.

\subsection{Support for Memory and ADL}

One of the main effects of mild dementia involves the impairment and loss of short-term memory. Solutions to overcome this shortcoming are usually called "cognitive orthotics" and range from medication reminders to devices that enable users to find misplaced objects. The use of memory aids has been a successful way to provide reminders to individuals with memory problems 
[15]. In parallel, daily routines are not only sacred, but a necessity to slow down the progress of the disease.

Memory problems often lead to the misplacement of objects and forgetting to perform daily activities (taking prescribed medication, eating, washing, and arranged appointments) [10]. This often leads to experiencing stress and frustration. Assistive devices have been developed to assist patients with $\mathrm{AD}$ in complying with their daily tasks and dealing with memory problems (see [2]). These include object locator devices through a process of object tagging; clocks and calendars displaying date and time; and reminders that alert people at pre-determined times.

Our aim is to partially automate routine identification and share the load among close people (family and friends, closest friends).

\subsection{Support for Social Contact and Company}

The progressive decline of short-term memory and cognitive function of $\mathrm{AD}$ patients result in a loss of communication skills. People usually seem to not be listening and are sometimes unable to follow a conversation and answer accordingly. Communication aids can therefore be used to facilitate and encourage communication with others. Two main areas are surveyed in this section: reminiscing therapy and in-situ communication support.

The goal of reminiscing therapy is to promote shared experiences between patients and caregivers. The load is placed on caregivers, as they have to constantly come up with discussion topics, which is time consuming and has low deployment rates. Communications aids were developed to improve this process. CIRCA [9] assists people with dementia by being able to select from a variety of videos and music via a touch screen interface contents that are likely to prompt memories of the PwD. The device provided a naturalistic conversation experience by removing pressure from the caregivers to continually prompt and empowered the person with dementia to participate on a more equitable basis [12]. Additional research shows that reminiscing therapy aimed at stimulating feelings and memories (e.g. videos, pictures, archives life story books and life reviews) are valuable and beneficial enhancing well-being. This is supported by other findings, which show that unfamiliar items to people with dementia are problematic and misunderstood [18].

The concept of using a memory wallet [7] with relevant selfprompt factual information during conversations with familiar partners has also shown to improve the conversations by making more actual factual statements. In this project, memory wallets contained 30 pictures and sentences about familiar people, places, and events that each participant had difficulty remembering. Results supported the utility of a memory wallet for persons with dementia and provided preliminary evidence that they improve conversations with familiar conversational partners. More recent personalized multimedia contents have been used to improve reminiscence with proven effectiveness [8].

Our approach tries to automate the process of memory gathering along with maintaining its relevance to the specific PwD.

\subsection{Enhanced Safety and Perceived Safety}

This is an unmet need that may jeopardize the remaining areas of action as the fear and lack of confidence felt by both patients and caregivers generally leads to anxiety and diminishing social contact and healthy daily activities. When inside the house, activity monitors and sensors have been used to understand the patient's condition and ensure safety. These devices include activity monitors; alarm and pager units; fall detectors; flood detectors; water temperature monitors; smoke, gas, carbon monoxide, and extreme temperature detectors; and lighting and wandering devices.

Outside the house, this problematic gains higher relevance. A common feature of $\mathrm{AD}$ is wandering. An example of a wandering device is the Digital Angel wandering device [14]. This device is composed of a watch and a pager unit with GPS. When a patient goes out of a pre-designated area, the system alerts caregivers via e-mail. iWander's [17] goal was to identify wandering behaviors on people with mild dementia using GPS. When abnormal behavior is detected several actions can take place: give directions to navigate the user to their home, sending notifications to the caregiver containing the location of the patient, establishing a line of communication between the patient-caregiver and performing a party call between the caregiver-patient and patient's local 911.

With the current smartphone devices, understanding the whereabouts and activity of a person is easy enough. Being able to characterize and infer events from a person's daily activities will also allow us to predict stressful or harmful situations before they happen being then able to act to increase the safety of the PwD.

\subsection{Friendsourcing}

Friendsourcing is defined as a form of crowdsourcing aimed at collecting accurate information available only to a small, socially-connected group of individuals [4]. These systems prevail over their anonymous and impersonal crowd counterparts when knowledge about a person or a context is required. More to it, when in quest for information, people already refer to their online social networks (e.g. Facebook or Twitter) to receive fast and trustworthy responses. This breed of human-powered access technology can be characterized in Bigham et al.'s framework [6] as one where motivation is intrinsic, work is unpaid, workers are non-experts and the reliability is undetermined.

Asking questions to the user's own social network has been explored in the context of accessibility; enabling blind people to ask questions about photos taken to their peers is one example [3].

There is still a paucity of usages of friendsourcing for PwD. We aim to change that as a way to improve our cognitive aid but also to strengthen and maintain the presence of a larger group of people around the PwD.

\section{A FRIENDSOURCED COGNITIVE AID}

Smartphones, powerful as they are in sensing, communication and processing, have been the target for the deployment of recent tools for PwD. Preset or remote reminders, GPS trackers, media collectors are easily deployed with or without user intervention. However, achieving a meaningful cognitive prosthesis that is able to bridge the lack of memories presented by PwD is farther away. People are different and the relevancy of their life events is something that not always can be assessed automatically.

The backbone of our system is a cognitive prosthesis automatically enriched with mobile and web data that is able to infer relevant life events and routines. The novelty is on the automatic prompting for knowledge validation and enrichment. This is achieved through a mainstream social network (at the time, Facebook) providing the required convenience for those involved.

\subsection{System Design}

The system is divided into three main conceptual parts: the mobile application, the knowledge base and the social and web component (Figure 1). The mobile application gathers data from 
its owner daily usage. This includes GPS tracks, motion data, call and message logs, contacts, photos and videos taken, ambient sound, among possible others. All data is annotated with timestamps. This data is sampled and sent to the server with few or no processing on the phone. The different types of data can be collected or not depending on the user's parameterization.

On the server, the data is stored into a private database. The Event Inference component is dedicated to identifying relevant life events from the raw data collected. It is based on previously trained classifiers working with the different types of data provided (one example is the identification of a life event with position shifts - when a user stays in the same place for a certain amount of time an event is created). These events are stored in a dedicated RDF-based semantic network, the knowledge base. Each Life Event is composed by a Place, Time, People and Media. By turn, each of those can be enriched with Media. Reasoning over this knowledge allows for the detection of routines.

Still, the data therein is restricted to the limits of what automatic tools can provide. The social and web component seeks to provide relevance and personalization to the knowledge in the cognitive prosthesis. The data is pre-enriched with available web services (getting place names from GPS data - e.g., using Google Places or generic photos from an address - e.g., using Flickr). With this data, more concrete questions can be made to the private social network to validate it, or get more relevant knowledge about it.

Figure 2 shows one example where the system automatically prompts the support network to identify where the PwD was during that morning. Several other types of questions are posed depending on the type of data available about an identified event (e.g. posting a photo to be identified and people therein identified, asking for a photo of a place or someone, asking to rate the relevancy of an event or a place, asking to tag a place). Members of the support network can add events and data without a prompt from the system. This enables the addition of events that are not automatically detected but also to populate the knowledge base with older life events. Such a capability supports the creation of life photo albums. Although not created for that purpose, the contents available in this site are by themselves a suitable tool to foster the relationship between the PwD and the support network.

\subsection{Applications}

The cognitive prosthesis enables bridging the unmet needs of PwD in several ways. Some current scenarios are presented.

Reminiscence Therapy Tool. This tool builds on the retrieved knowledge to show to the user and caregivers the resume of a day, week, month, year, or life. Also, it allows for cross-consultation of Places, People and Time. The advances beyond the state of the art are in presenting broad but still relevant memories.

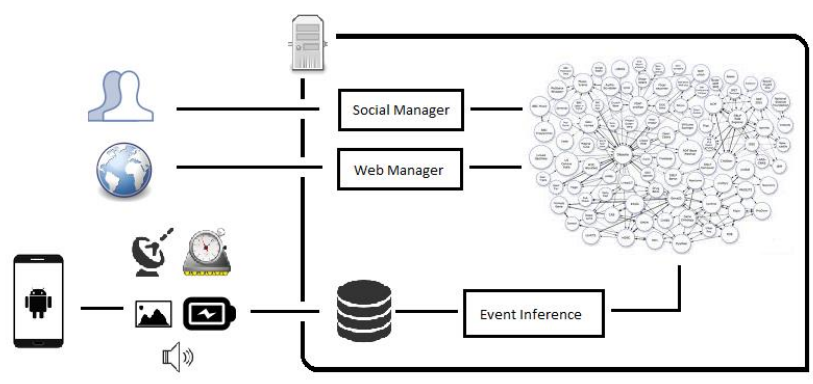

Figure 1. High-level system design.

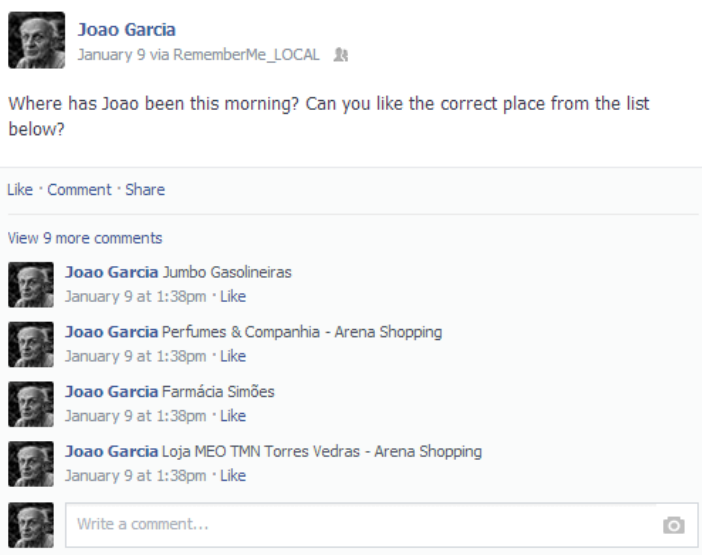

Figure 2. A question posed automatically in the PwD's private group. The members answer by using the "like" mechanism.

In-situ Communication Aid. Being able to associate memories with Places and People, and reference Times, the user itself can be prompted with useful information automatically. This tool presents information to the user depending on his context.

Daily Routines and Reminders. By identifying patterns, we are able to assess and prompt the user to follow habits. Also, explicitly we can allow the setting of reminders through the private social network easily deploying a remote pager.

\subsection{PRELIMINARY ASSESSMENT}

This project is being developed in collaboration with an association for PwD. An interview was performed with the person responsible for the psychology and formation body of the association. This department is devoted to give support to people with Alzheimer but also to aid and give formation to caregivers either formal or informal ones. The interview was performed with a particular focus on the automatic gathering, identification, storage of life events and on the friendsourcing component. After a brief explanation of our approach, its potentialities were presented in the form of usage scenarios. This enabled the person to grasp the idea of all the people and information involved in the usage of such system. The main considerations retrieved were:

Towards more balanced tools. The staff of the association showed to be knowledgeable about monitoring and reminiscence aids available. However, when asked about their success, there was a paucity of examples or references. The tools that have been put to use were either too troublesome for the caregivers or misaligned with the needs of PwD. The interviewee mentioned that the differences between PwD and the evolution of the condition and associated needs required tools that could adapt to better suit their users. When confronted with our approach, she identified the potential to do so, stating that it is essential to have a tool that can balance the load between the PwD, that can be sometimes capable to be the main parameterizer of the tool and provide data without help and the caregivers, that can have a major role when the cognitive abilities become compromised.

Engaging a larger support network. One concern stated upfront was how quick the number of informal caregivers around a PwD decreases with time. People really want to stay in touch but with their busy lives and the painful experience of watching in the first row a friend deteriorate his cognitive abilities.....it gets common place to have an excuse to avoid having a lunch or going for a coffee. This concern was first presented as a negative 
and damaging issue towards our approach. If the PwD support network is quickly restricted to one or two people, the load will also be on them, which renders your system useless. Although our system is designed to make the creation of reminders, recognition of routines and managing alerts easily enough, this is indeed a concern that can hinder the success of the approach. However, the psychologist stated that, having a digital medium that is lightweight might be the required tool to allow for people to maintain connected and engaged in helping the PwD. Out of sight, away from the heart. This system may change that.

The right for privacy. One pressing issue is the one of privacy. In the target group the variations in consciousness make it difficult to assess when the main user is able to decide his privacy settings. On the other hand, no one else can decide on his/her behalf exception made if legal responsibility over the person is attained and this only happens in later stages of dementia. As with current GPS systems, this can be solved with a conscious action to start using those systems as they are, but a conservative stance needs to be employed (e.g., caution with recording audio, video, voice calls and messages). Another issue with our approach is that some of these contents are shared among a support network that aims to go beyond the closer family members to be most effective. This is powerful but different levels of privacy need to be established. One may allow for a close family member to know where one is all the time but not everyone. This comment calls out for having several groups per PwD instead of a single one.

The challenge of presenting contents. The information collected makes place for a cognitive prosthesis that will eventually be more complete than the one of the person who lived those moments. A naive approach for reminiscence therapy would be to present information to the $\mathrm{PwD}$ despite their knowledge about those particular events in their lives. It became clear in this elicitation that selecting which information should or should not be presented is a challenge by itself. Showing something to a person that no longer remembers it can be by itself a cause of depression. Imagine you are showing someone who is her son when she no longer remembers him. This type of confrontation can be daunting and have opposite effects to what is desired.

\section{CONCLUSIONS}

Digital support tools for people with dementia range from simple reminders to sophisticated cognitive prosthesis. There is a pervasive trend for these tools to be designed around the PwD and a very limited set of caregivers. Although this is understandable given the paucity of people around a PwD as time goes by, our approach settles over the idea that by accounting for a larger support network, these tools can be more effective. Further, the relationships between the PwD and these multi-level support networks (family, close friends, neighbors, restaurant owners, officers,..), which are often otherwise rendered inexistent, can be maintained if the different stakeholders feel engaged. Future steps include refining the way in which life events are identified and enriched along with tuning the process of friendsourcing to make it more agile and useful. We envision a longitudinal study with volunteer families and PwD to assess the benefits of this proposal.

\section{ACKNOWLEDGMENTS}

This work was supported by FCT through funding of SiTECoSE project,.PTDC/EIA-EIA/117058/2010, and LaSIGE Strategic Project, PEst-OE/EEI/UI0408/2014. We also thank Alzheimer Portugal for their support in the project.

\section{REFERENCES}

[1] Alzheimer's Disease International (ADI) (2009) World Alzheimer's report 2009, executive summary, p. 2.

[2] Armstrong, N., et al. (2010). Using smartphones to address the needs of persons with Alzheimer's disease. Annals of Telecommunications, 65(9), 485-495.

[3] Brady, E. L., et al. (2013). Investigating the appropriateness of social network question asking as a resource for blind users. In Proceedings of the CSCW (pp. 1225-1236). ACM.

[4] Bernstein, M. S., et al. (2010). Personalization via friendsourcing. ACM (TOCHI), 17(2), 6.

[5] Bigham, J. P., et al. (2010). Vizwiz: nearly real-time answers to visual questions. In Proceedings of UIST (pp. 333-342).

[6] Bigham, J. P., et al., The Design of Human-Powered Access Technology. The Proceedings of the $13^{\text {th }}$ International ACM SIGACCESS Conference on Computers and Accessability, ASSETS '11, Dundee, Scotland, UK. ACM 978-1-45030920-2.

[7] Bourgeois, Michelle S. "Evaluating memory wallets in conversations with persons with dementia." Journal of Speech, Language and Hearing Research 35.6 (1992): 1344.

[8] Buchanan, J. A., et al. (2011). The role of behavior analysis in the rehabilitation of persons with dementia. Behavior therapy, 42(1), 9-21.

[9] CIRCA: Computer Interactive Reminiscence and Conversation Aid (2001). http://www.computing.dundee.ac.uk/projects/circa/.

[10] Davies RJ et al (2008) A user driven approach to develop a cognitive prosthetic to address the unmet needs of people with mild dementia. Pervasive Mobile Comput 5(3):253-267

[11] C. Dobbins, et al., Creating human digital memories with the aid of pervasive mobile devices, Pervasive and Mobile Computing (2013),

[12] Gowans G, et al. (2004) Designing a multimedia conversation aid for reminiscence therapy in dementia care environments. In Proc of CHI, pp 825-836

[13] Logsdon, R. G.,et al. . (1999). Quality of life in Alzheimer's disease: patient and caregiver reports. Journal of Mental Health and Aging, 5, 21-32.

[14] Parnes PB (2009) GPS: technology and Alzheimer's disease. Novel use for an existing technology. Alzheimer's article, Third Age, 2008.

[15] Pollack M, et al. (2003) Autominder: an intelligent cognitive orthotic system for people with memory impairment. Robot Auton Syst 44(3-4):273-282

[16] Reinersmann, A., et al. (2007) Review of ICT-based services for identified unmet needs in people with dementia. Ageing Research Reviews, 6(3): 223-246.

[17] Sposaro, F., et al., "iWander: An Android application for dementia patients," Engineering in Medicine and Biology Society (EMBC), IEEE , pp.3875-3878, 2010

[18] Swann, J. Preserving memories: using reminiscence techniques. Nursing \& Residential Care, 2008, Vol 10 (12). 